\title{
Discrepancia estructural del disco y cápsula articular de la ATM en resonancia nuclear magnética. Revisión de la literatura
}

\begin{abstract}
Structural discrepancy of the disc and articular capsule of the TMJ on magnetic resonance imaging. Literature review
\end{abstract}

Anne-Marie Kusch 1,2,a,b, Abell Sovero Gaspar ${ }^{1, a, b}$

\section{RESUMEN}

La articulación temporomandibular (ATM) es considerada una articulación del tipo diartrosis bicondílea. La evaluación imagenológica comprende una parte fundamental en su evaluación, la que debe abarcar tanto las características óseas como los tejidos blandos y la relación entre ellos. La resonancia nuclear magnética (RNM) representa el gold standard para el estudio de tejidos blandos, sin embargo, hasta ahora su mayor aplicación continúa siendo la visualización del disco articular. Es por esto, que el presente artículo tuvo como objetivo señalar la información disponible en la literatura con respecto a la visualización de la cápsula articular en RNM y evaluarla como una estructura independiente.

Palabras-clave: Articulación temporomandibular, disco de la articulación temporomandibular, imagen por resonancia magnética.

\section{SUMMARY}

The temporomandibular joint (TMJ) is considered a bicondylar diarthrosis joint. Imaging evaluation comprises a fundamental part of its evaluation, which must include both bone characteristics as well as soft tissues and the relationship between them. Magnetic resonance imaging (MRI) represents the gold standard for the study of soft tissues, however, until now its greatest application continues to be the visualization of the articular disc. This is why the present article aimed to point out the available information in the literature regarding the visualization of the joint capsule in MRI as an independent structure.

Keywords: temporomandibular joint, articular disc, magnetic resonance imaging.

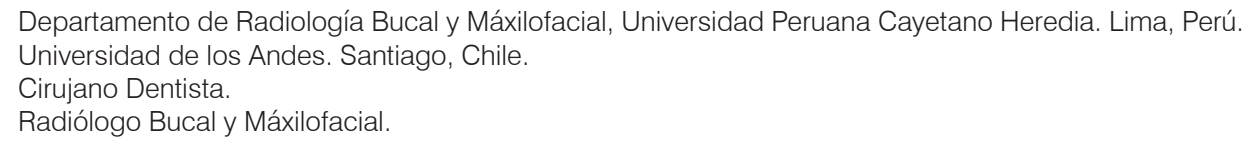




\section{INTRODUCCIÓN}

La articulación temporomandibular (ATM) es considerada una articulación del tipo diartrosis debido a su capacidad para realizar movimientos de traslación y rotación, y bicondílea ya que articula dos superficies distintas. Difiere de otras articulaciones del organismo por estar recubierta por un tejido fibroso avascular o fibrocartílago en vez de cartílago hialino, las superficies óseas que articula (maxilar y mandíbula) poseen dientes y forman una sola unidad desde el punto de vista funcional ${ }^{(1)}$.

Dentro de las indicaciones establecidas para su estudio por medio de imágenes se encuentran tratamientos conservadores fallidos, incremento en sintomatología, historia de trauma, disfunción significativa, cambios importantes en la oclusión, anormalidades estructurales óseas, sospecha de infecciones, alteraciones sensoriales o motoras ${ }^{(2)}$. Su correcta visualización frente a casos de patología, planificación de procedimientos quirúrgicos o disfunciones resulta fundamental para el proceso diagnóstico ${ }^{(3-6)}$. Para evaluar su integridad, debe abarcarse tanto las características morfológicas óseas o tejidos duros (cóndilo mandibular, fosa y tubérculo articular del hueso temporal), así como los tejidos blandos (disco articular, ligamentos y cápsula articular) y la relación entre ellas, tejidos circundantes musculares que la rodean no deben obviarse, especialmente aquellos que poseen una íntima relación como lo es el músculo pterigoideo lateral (vientre superior) ${ }^{(7)}$. Este análisis permitirá confirmar la extensión o progresión de alguna disfunción o enfermedad si existiera, y también documentar y evaluar los efectos de algún tratamiento ya establecido ${ }^{(8)}$. Para evaluación de tejidos duros, las técnicas de visualización en $2 \mathrm{D}$ tienen serias limitaciones con respecto a la superposición de estructuras anatómicas principalmente al cráneo y al arco cigomático, los que obstaculizan la visualización del cóndilo mandibular y de la fosa glenoidea. Es por esto, que las técnicas antiguas (radiografía panorámica y proyección transcraneal) han sido reemplazadas por tomografía computarizada (TC $)^{(4)}$. Actualmente, debido a que posee una menor dosis de radiación es que se aplica Tomografía Computarizada de Haz Cónico (TCHC) con una precisión diagnóstica en cambios óseos similar a la TC. ${ }^{(9-12)}$ Sin embargo, ninguna de estas dos modalidades brindan correcta visualización de tejidos blandos, por lo que otros métodos diagnósticos deben aplicarse ${ }^{(9,13)}$. Con respecto a tejidos blandos, se utilizan diversas tecnologías como la artrografía, indicada para la evaluación de la posición del disco, perforaciones y adhesiones discales, requiere de la aplicación de contraste intracapsular ${ }^{(8)}$; por lo que resulta difícil de realizar ${ }^{(14)}$. La ultrasonografía, permite evaluar tejidos blandos tanto en movimientos como estáticos (boca abierta y cerrada), es una herramienta diagnóstica económica, transportable, de menor tamaño y requiere de menos infraestructura, brindando mayor comodidad al paciente, pero operador-dependiente $(4,8)$. La RNM entrega información anatómica y funcional tanto de tejidos duros como blandos ${ }^{(7,14)}$. Pese a que no tiene gran precisión para visualizar tejido óseo cortical, la mayoría de patologías óseas son pesquisadas mediante este examen imagenológico ${ }^{(13,14)}$. Protocolos actuales, recomiendan la combinación de exámenes auxiliares imagenológicos para el estudio de tejidos duros con TCHC como para tejidos blandos con RNM, cuando resulta necesario para el diagnóstico ${ }^{(6)}$. Son pocos los estudios y revisiones publicadas en la literatura enfocadas en la visualización de estas estructuras de tejidos blandos definiéndolas como entidades independientes ${ }^{(15-18)}$. la mayoría se enfoca en el estudio del disco articular.

El objetivo de esta revisión de la literatura fue presentar la información disponible en la literatura con respecto a si la cápsula articular de la ATM puede ser visualizada y evaluada en RNM o no y si ésta puede ser distinguida del disco articular o no.

\section{RNM en ATM}

Es el examen imagenológico considerado como gold-standard hasta el día de hoy ${ }^{(19)}$ posee una resolución espacial y de contraste excelente y no provee radiación ionizante ${ }^{(4,19)}$. Toda la estructura de la ATM puede ser claramente distinguida por el reconocimiento de las distintas intensidades de señal tanto de estructuras óseas, cartilaginosas, musculares así como el líquido sinovial ${ }^{(20)}$. Convirtiéndola en la técnica imagenológica sugerida para el análisis del disco articular de la ATM y de las estructuras anatómicas circundantes tanto en estado normal como ante la presencia de patologías ${ }^{(21,22)}$.

\section{Anatomía de tejidos blandos en RNM}

Todas las técnicas imagenológicas descritas previamente para la visualización y evaluación de tejidos 
blandos de la ATM no están exentas de dificultades. El desafío radica en la visualización correcta de las estructuras en esta área, ya que son pequeñas en relación al grosor de corte, además cualquier mínima variación en la alineación del plano pueden alterar la forma en que las imágenes se observan y por último, las estructuras de interés tanto en RNM como en TC normalmente no se ven en su totalidad, sino que para observarlas se deben ver cortes consecutivos ${ }^{(23)}$. Además la ATM se encuentra próxima a estructuras anatómicas como lo son las celdillas mastoideas, estructuras auditivas y al hueso temporal, pudiendo llevar a confusiones ${ }^{(19)}$. El protocolo sugerido incluye planos oblicuos sagitales, coronales perpendiculares y paralelos al eje mayor de la cabeza condilar (figura 1), con un grosor de corte menor o igual a $3 \mathrm{~mm}$ y con una antena de superficie dual para poder captar la señal de ambas ATMs ${ }^{(24-26)}$. Se recomienda la aplicación de secuencias ponderadas T1, T2 con supresión grasa y densidad protónica tanto en boca abierta como cerrada ${ }^{(26)}$. La aplicación de un medio de contraste como el gadolinio se emplea para determinar la presencia de artropatías en ciertos pacientes ${ }^{(27)}$. Poco difundido es el estudio dinámico, el que se utiliza principalmente para la evaluación del disco articular y su relación con las estructuras óseas vecinas ${ }^{(26)}$.

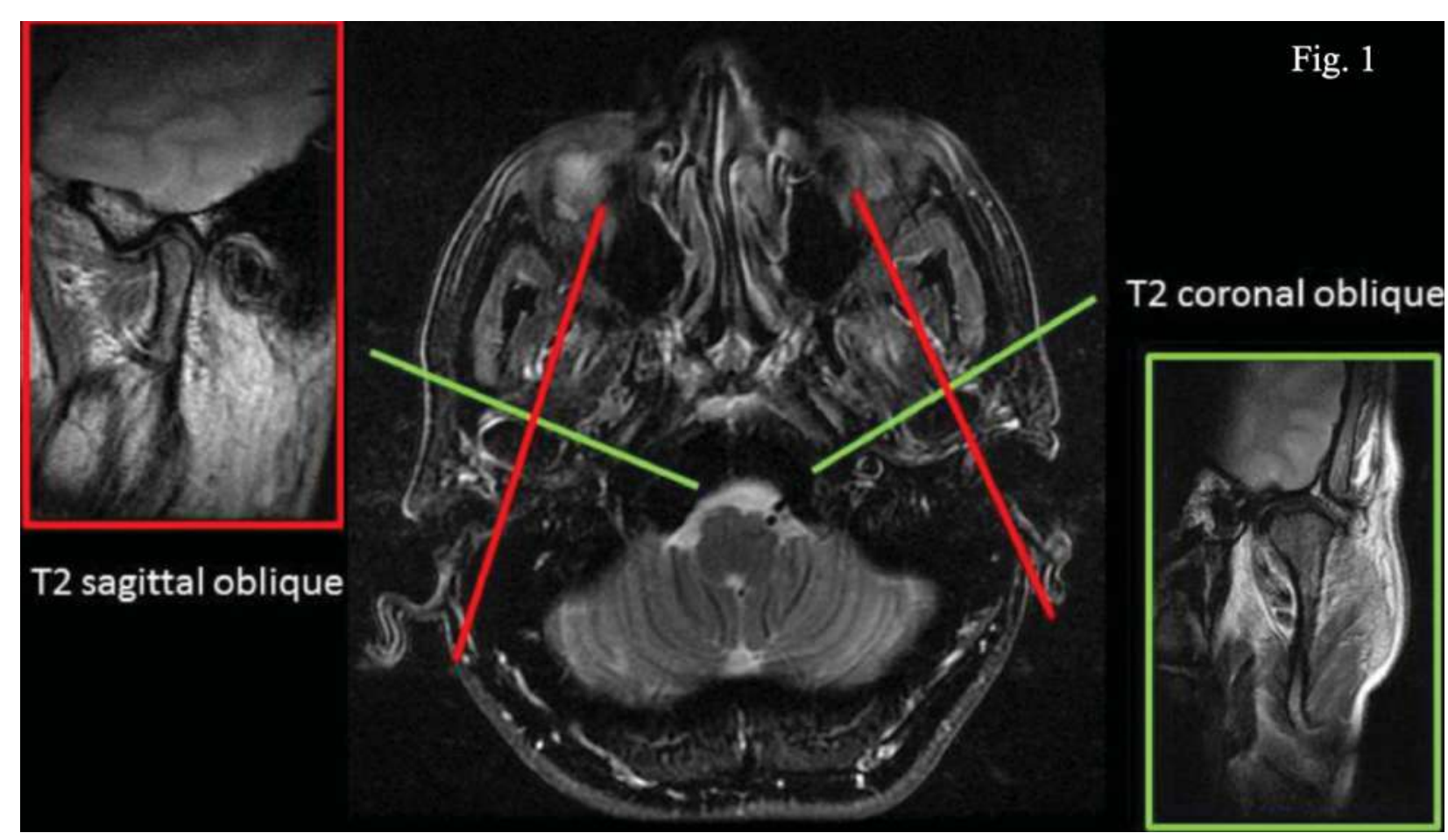

Figura 1. Ilustración de cortes en RNM de ambas ATM en T2: sagital oblicua y coronal oblicua ${ }^{(28)}$.

Ampliamente divulgado resulta la aplicación de la RNM para el estudio del disco articular no tan sólo su morfología sino también su dinámica, existiendo así mismo protocolos bien establecidos para ello ${ }^{(26,29,30)}$. El disco articular de la ATM está formado por tejido conectivo fibroso, en cortes sagitales de RNM se observa como una estructura bicóncava con una señal homogénea de baja intensidad o hipointensa en su zona anterior, así como la intermedia y la posterior se observa como leve hiperintensidad en condiciones normales o de salud y la zona bilaminar posee una se- ñal intermedia en T1 ponderado. ${ }^{(8,26)}$ la porción superior de esta zona bilaminar es rica en fibras elásticas y la inferior en fibras colágenas, ambas separadas por tejido areolar laxo. ${ }^{(1)}$ Los tejidos de la zona bilaminar y del músculo pterigoideo lateral presentaran una señal moderada en imágenes T2 ponderadas, mucho más bajas que en $\mathrm{T} 1$ ponderada. Se ha demostrado que cortes parasagitales así como paracoronales (figura 2), en T1 ponderado y en difusión protónica ponderada en boca cerrada dan la mejor imagen para evaluar la anatomía de la ATM y no así T2 ponderada ${ }^{(28)}$. 

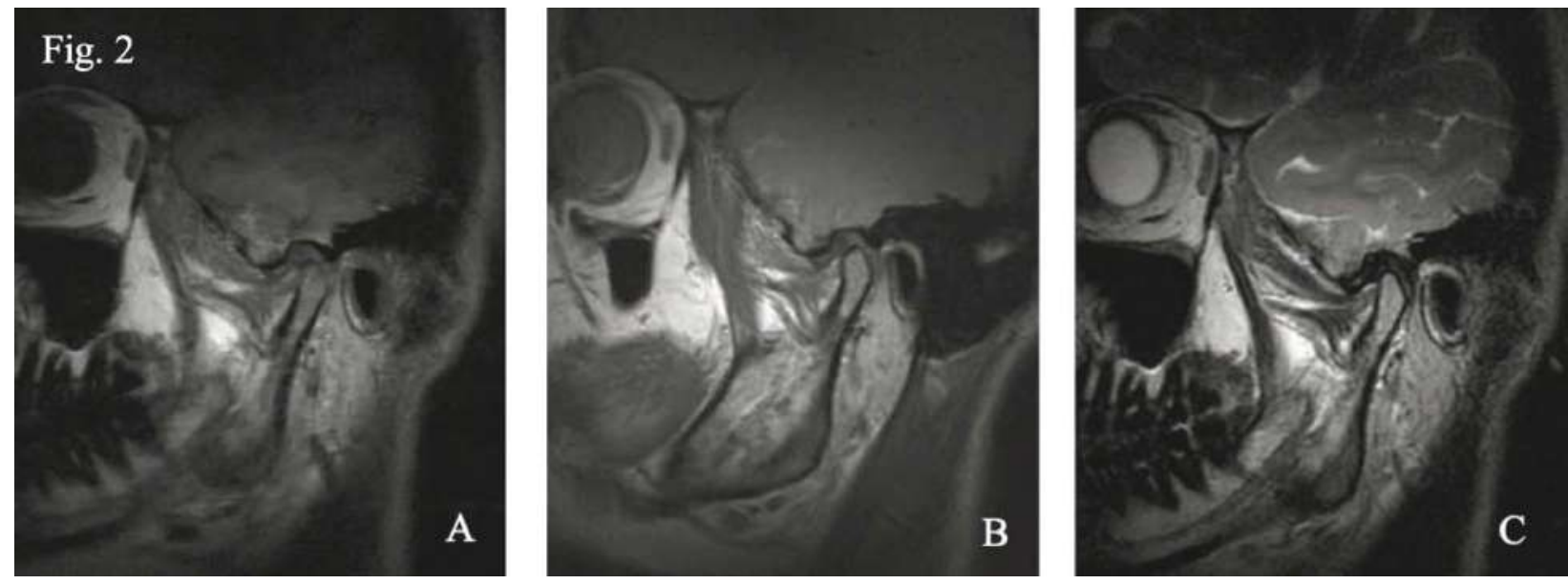

Figura 2. A: Ejemplos de vista parasagital de ATM en boca cerrada. A: imagen ponderada en T1.B: imagen ponderada en difusión protónica. C: imagen ponderada en T2. Se evidencia con mayor facilidad los distintos componentes anatómicos tanto en la imagen A como B si se compara con la $\mathrm{C}^{(31)}$.

El disco articular se une en ambos extremos a la cápsula articular que lo rodea, dividiendo a la articulación en dos compartimientos: supradiscal y infradiscal ${ }^{(25)}$. El aparato ligamentoso de la articulación está compuesto por la cápsula y ligamentos. Dentro de los ligamentos, se encuentra el ligamento temporomandibular o lateral externo asegura de manera lateral a la cápsula articular y se conoce como el principal refuerzo. Los ligamentos esfenomaxilar y estilomaxilar tienen un rol accesorio, así como el mandíbulomaleolar, ligamento fibroelástico el que comparte origen embriológico con los huesos martillo y yunque, pudiendo explicar el hecho de que en cuadros de disfunción temporomandibular éstos se acompañen con cuadros de sintomatología auditiva. Sin embargo, resulta complejo en cortes sagitales de disco articular determinar si este ligamento es un elemento independiente o bien una extensión lateral del ligamento esfenomaxilar. Todos estos elementos se componen de tejido conectivo compacto con predominio de fibras colágenas. Ambos compartimientos discales (supra e infradiscal) están recubiertos interiormente por las membranas sinoviales, éstas están ubicadas sólo en la periferia sin tener relación alguna con el disco articular ${ }^{(1)}$, estos presentan una intensidad de señal intermedia ${ }^{(15)}$. La cápsula es una estructura fibrosa laxa que rodea a toda la articulación excepto en su cara ánteromedial debido a que en este punto se encuentra la fusión de las fibras tendinosas del haz superior del músculo pterigoideo lateral con el disco articular, ${ }^{(1)}$ lo que muchas veces puede dar imagen de doble disco articular en RNM ${ }^{(16)}$.
Con respecto a esta última estructura, resulta controversial si se observa o no en RNM. La mayoría de estudios no especifican ${ }^{(8,25,32)}$, pocos afirman que no (24), otros sólo frente a casos de patologías o distención por efusión ${ }^{(23,28,29,33)}$, siendo muy pocos los que afirman que sí puede verse ${ }^{(15,17)}$ (figura 3 ), otros a raíz de casos de fractura como Kim et al. ${ }^{(34)}$, sólo mencionan que se observa como una línea oblicua (figura 4), pero pero no especifican dónde se encuentra; Sun y Wan mencionan su ruptura y presentación como una masa expansible al canal auditivo ${ }^{(18)}$. Sin embargo, ninguno especifica cómo es que se ve. Sakhavalkar et al. ${ }^{(35)}$, en el año 2016 publicaron un estudio evaluando y midiendo el ancho de la cápsula articular, basados en parámetros indirectos, pero tampoco especifican cómo se observa. 

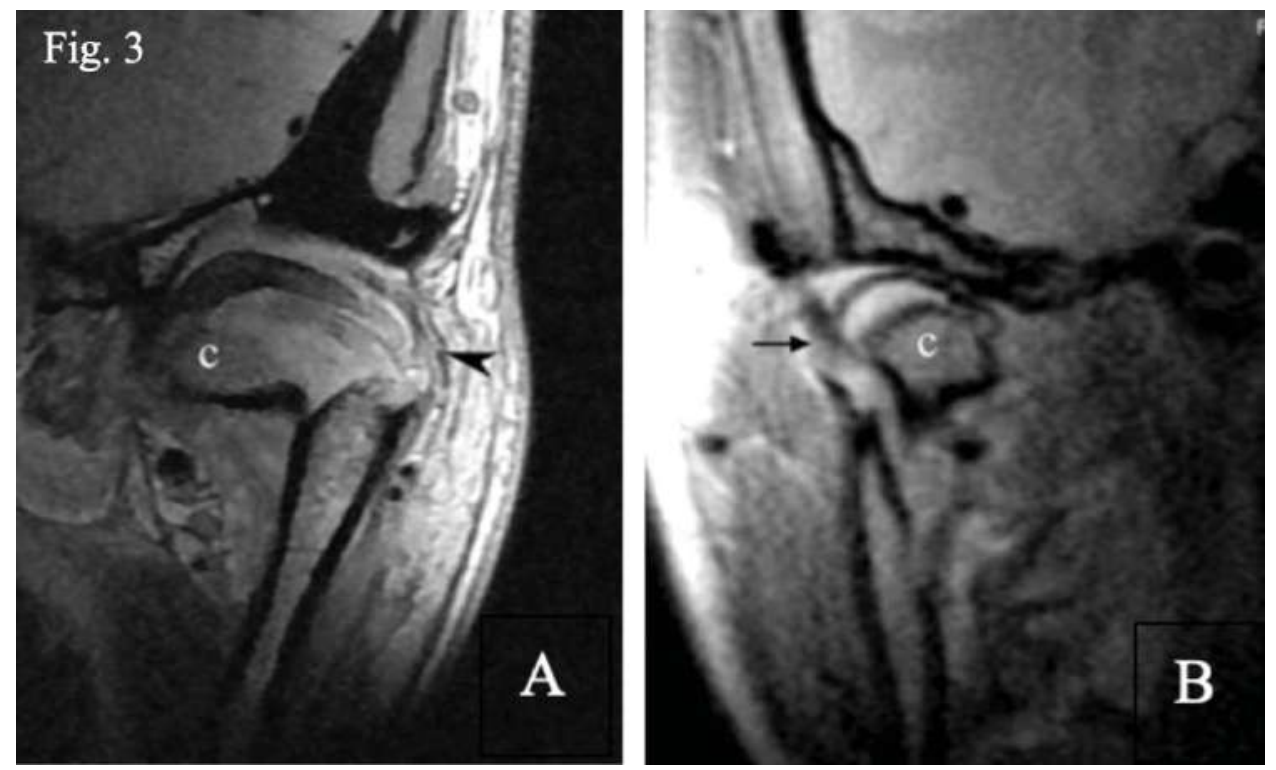

Figura 3. Ambas son imágenes ponderadas con atenuación protónica en vista coronal. En la imagen A se menciona que en (c) se ve un fragmento de fractura condilar y una imagen bien definida de la cápsula articular (cabeza de flecha). La imagen B grafica la imagen bien definida de la cápsula articular (flecha negra) y en (c) la fractura del cóndilo en la zona medial de la rama mandibular ${ }^{(17)}$.

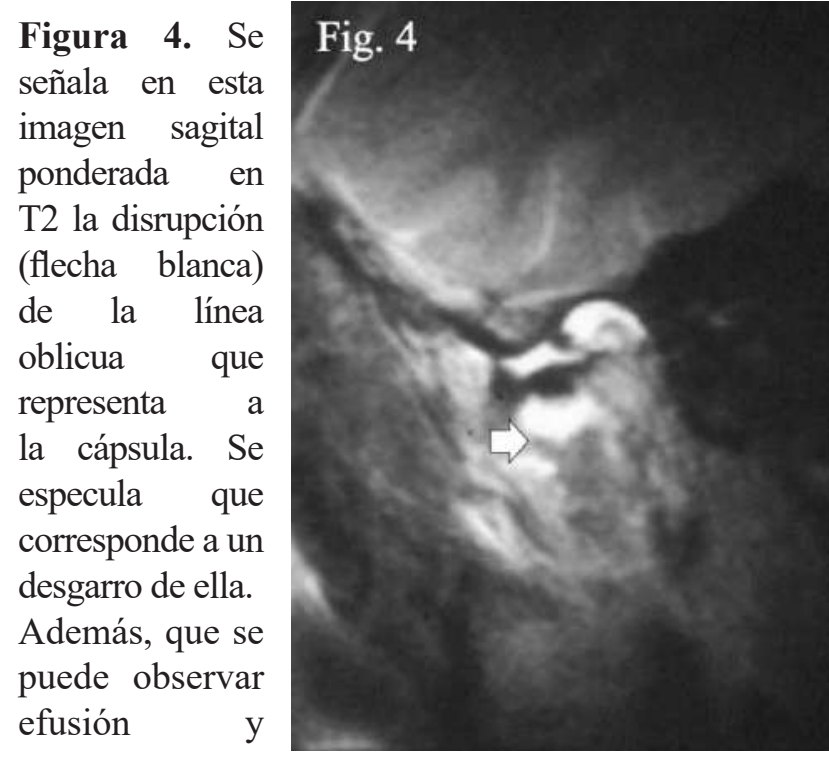

hamartrosis en ambos compartimientos articulares ${ }^{(34)}$.

Con este artículo se quiere postular el hecho de que el disco articular muchas veces es confundido con la cápsula articular cuando se evalúa en RNM y no puede determinarse como una estructura independiente. Contraste es definido como la diferencia visual entre las distintas sombras negras, blancas y grises contenidas en una misma imagen. Éste puede alterar la densidad advertida entre dos estructuras adyacentes, ya sea por la propia composición de estas estructuras o por elementos externos a ellas ${ }^{\left({ }^{36)} \text {. Todo }\right.}$ diagnóstico imagenológico debe demostrar contraste entre características anatómicas normales y patológi- cas, sin esto resulta inviable identificar o pesquisar anormalidades $^{(37)}$. En RNM, el contraste entre dos distintos tejidos depende de las propiedades inherentes o intrínsecas de estos y de los parámetros secuenciales de pulso o extrínsecos determinados por el usuario ${ }^{(38)}$. Dentro de las primeras se encuentra la secuencia T1, T2, la densidad protónica, flujo y del coeficiente aparente de difusión, y dentro de las segundas el tiempo de repetición, tiempo echo, valor b, factor turbo, ángulo flip y tiempo de inversión ${ }^{(37)}$; se ha postulado que los dos primeros son los que más afectan la apariencia de los tejidos. Cabe destacar, que sólo una característica en la composición puede resultar determinante en la apariencia de un tejido en una imagen, independiente del intento de contraste brindado por la secuencia de pulso seleccionada (38). Uno de los desafíos que supone la evaluación de tejidos fibrocartilaginosos de la ATM en RNM es que éstos tienen un corto $\mathrm{T} 2$ el que cae abruptamente si se compara con el cartílago hialino ${ }^{(39)}$, debido a que los equipos no resultan sensibles a estos componentes, dando una señal de intensidad baja o inclusive cero en todas las secuencias; traduciéndose finalmente en imágenes grises o negras. Esto resultaría útil para evidenciar anormalidades que posean una alta señal ${ }^{(40)}$, sin embargo, genera confusión cuando se quiere individualizar estructuras anatómicas próximas, especialmente cuando éstas comparten la misma composición. ${ }^{(38)}$ Una mejora a esta técnica estaría dada por la aplicación de tiempos eco ultra cortos, el que preservaría la señal del fibrocartílago y minimiza los artefactos ${ }^{(28,40)}$. Sin embargo, esta secuencia aún está reservada para investigaciones. 
Además, debe tenerse en consideración que hasta hoy la mayoría de los resonadores utilizados son de 1.5 tesla mientras que aquellos de mayor potencia de campo magnético brindan imágenes con mayor precisión diagnóstica ${ }^{(41)}$. Por eso, creemos que varios artículos no han logrado diferenciar estas estructuras por los factores inherentes del equipo resonador. Para graficar esto, en la imagen (figura 5) presentada por Orhan et al., en el año 2005 se puede ver claramente lo imposible que resulta diferenciar el disco de la cápsula articular ${ }^{(42)}$. A medida que la tecnología va evolucionando, autores como Wang, Yang y Yu en el año 2009 ya mencionan su visualización en T1 y con una densidad muy similar a la del disco articular particularmente cuando se observa en la reformación coronaria ${ }^{(17)}$. Luego ya para el año 2015 Hagenkord y Basel presentaron imágenes de la cápsula articular donde la definieron con una densidad similar a la de la cápsula, como fue comentado anteriormente ya que están conformadas por el mismo tejido ${ }^{(43)}$; así, con una imagen de archivo queremos demostrar que la imagen del disco articular puede ser diferenciada de la cápsula articular (figura 6).

Por último, debe considerarse lo descrito ya en el año 2012 por Shaefer et al. ${ }^{(44)}$ quiénes recalcaron

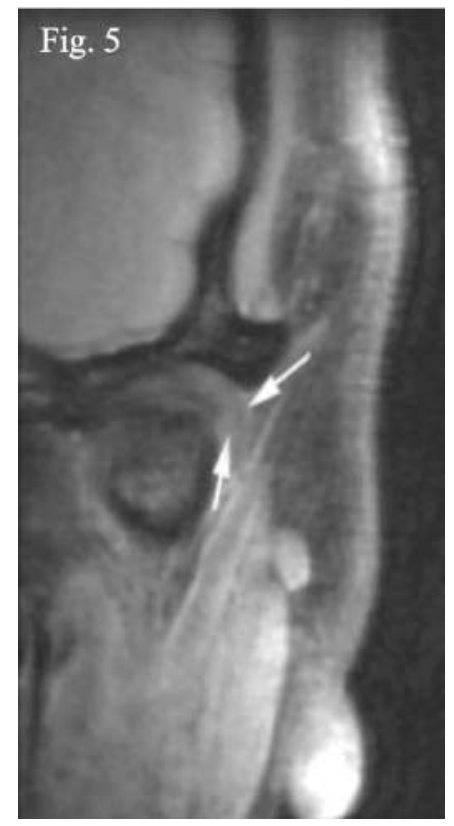

Figura 5. Reformación coronaria de RNM, donde se visualiza el desplazamiento lateral del disco articular (flechas blancas) ${ }^{(42)}$. la importancia del análisis de la RNM en cortes sagitales y coronales corregidos, métodos descritos anteriormente por Steenks et al., ${ }^{(45)}$ y Chen et al., ${ }^{(46)}$, ambos evidenciaron que la aplicación de parámetros funcionales podían mejorar la técnica para determinar el grado de desplazamiento discal. Esta modificación consiste en la observación del disco en cortes coronales paralelos a una línea perpendicular a la inclinación más posterior de la eminencia articular (figura 1).

\section{CONCLUSIONES}

Resulta particularmente complejo el distinguir la cápsula articular del disco. Aún a un especialista, le cuesta diferenciar los matices de grises entre un cartílago hialino y uno fibroso, los que tienen bajo contraste pudiendo fácilmente confundir ambas estructuras. Además, podríamos agregar que los cortes entregados pudieran no ser una fiel representación de las estructuras anatómicas de la articulación temporomandibular. Por esto último, resulta indispensable la entrega del volumen completo de la RNM o TCHC realizada para poder realizar un análisis exhaustivo en los cortes señalados en los protocolos.

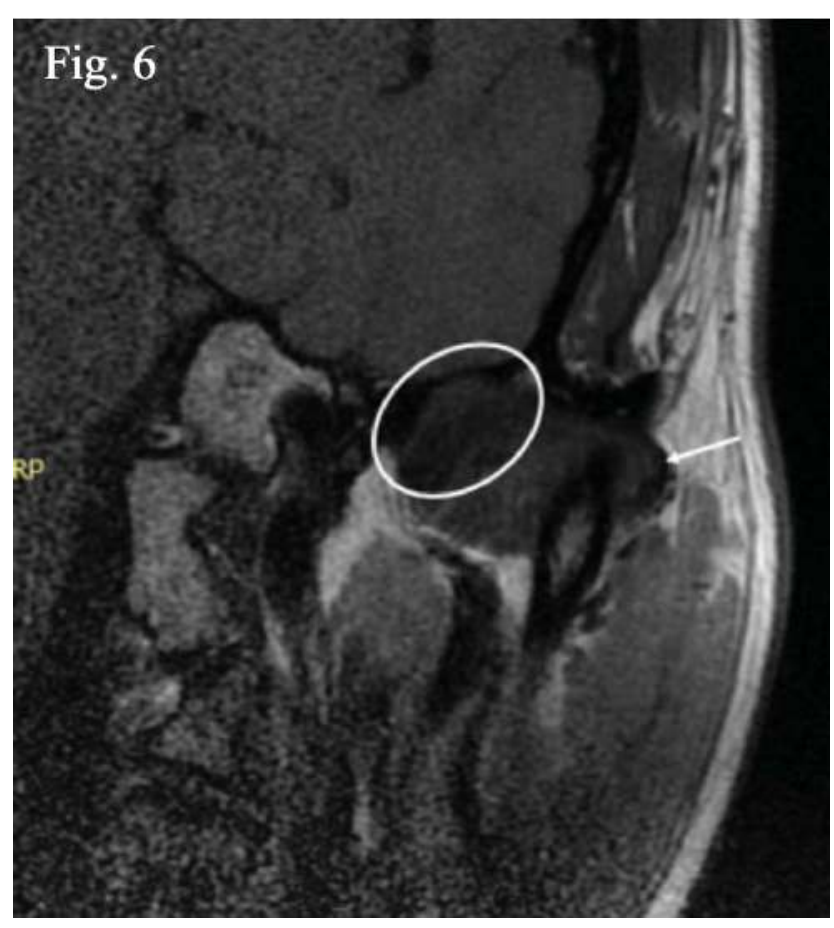

Figura 6. Imagen de archivo del Servicio de Radiología Bucal y Máxilofacial de UPCH. Reformación coronaria en RNM. Se señala la imagen del disco articular (óvalo) y la cápsula articular (flecha blanca), distinguibles una estructura anatómica de la otra. 


\section{Correspondencia:}

Abell Sovero Gaspar

Correo electronico: abell.sovero.g@upch.pe

\section{REFERENCIAS BIBLIOGRÁFICAS}

1. Manns A, Díaz G. Análisis morfofuncional de los componentes fisiológicos del sistema estomatognático. En: Manns A, Díaz G. Sistema Estomatognático. Santiago, Chile: Universidad de Chile; 1988. p. 9-18.

2. Perschbacher S. Disorders of the temporomandibular joint. In: White and Pharoah's Oral Radiology Principles and Interpretation. 8va ed. Toronto, Canada: Elsevier; 2019. p. 1633-726.

3. Brooks SL, Brand JW, Gibbs SJ, et al. Imaging of the temporomandibular joint: a position paper of the American Academy of Oral and Maxillofacial Radiology review. Oral Surg Oral Med Oral Pathol Oral Radiol Enod. 1997;83(5):609-18.

4. Baba I, Najmuddin M, Shah A, Yousuf A. TMJ imaging: a review. Int J Contem Med Resea. 2016;3(8):2253-6.

5. Ferreira LA, Grossmann E, Januzzi E, de Paula MVQ, Carvalho ACP. Diagnosis of temporomandibular joint disorders: indication of imaging exams. Braz $\mathrm{J}$ Otorhinolaryngol. 2016;82(3):341-52.

6. Schiffman E, Ohrbach R, Truelove E, Look J, Anderson G, Goulet JP, et al. International RDC/ TMD consortium network, international association for dental research; orofacial pain special interest group, international association for the study of pain. diagnostic criteria for temporomandibular disorders (DC/TMD) for clinical and research applications: recommendations of the international RDC/TMD consortium network* and orofacial pain special interest group. J Oral Facial Pain Headache. 2014;28:6-27.

7. Eren H, Eray Kolsuz M, Orhan K. An overall look for temporomandibular joint pathologies and imaging. Int J Orthop. 2015;2(6):452-61.

8. Bag AK, Gaddikeri S, Singhal A, Hardin S, Tran BD, Medina JA, et al. Imaging of the temporomandibular joint: an update. World J Radiol. 2014;6(8):567-82.

9. Honda K, Larheim TA, Maruhashi K, Matsumoto $\mathrm{K}$, Iwai K. Osseous abnormalities of the mandibular condyle: diagnostic reliability of cone beam computed tomography compared with helical computed tomography based on an autopsy material. Dentomaxillofac Radiol. 2006;35:152-7.

10. Zain-Alabdeen EH, Alsadhan RI. A comparative study of accuracy of detection of surface osseous changes in the temporomandibular joint using multidetector CT and cone beam CT. Dentomaxillofac Radiol. 2012;41:185-91.
11. Azevedo MQ de A, Barros RQ de A, Donato LFA, et al. Degenerative bone changes in TMJ assessed by cone beam computed tomography. RGO Rev Gaúch Odontol. 2016;64(2):171-8.

12. Shahidi S, Salehi P, Abedi P, Dehbozorgi M, Hamedani $\mathrm{S}$, Berahman N. Comparison of the bony changes of TMJ in patients with and without TMD complaints using CBCT. J Dent Shiraz. 2018;19(2):142-9.

13. Larheim TA, Abrahamsson AK, Kristensen M, Arvidsson LZ. Temporomandibular joint diagnostics using CBCT. Dentomaxillofac Radiol. 2015;44:20140235.

14. Concha G. Imágenes por resonancia magnética de la articulación temporomandibular. Rev HCUCh. 2007;18:121-30.

15. Vilanova JC, Barceló J, Puig J, Remollo S, Nicolau C, Bru C. Diagnostic imaging: magnetic resonance imaging, computed tomography, and ultrasound. Semin Ultrasound CT MRI. 2007;28(3):184-91.

16. Alomar X, Medrano J, Cabratosa J, et al. Anatomy of the temporomandibular joint. Semin Ultrasound CT MRI. 2007;28(3):170-83.

17. Wang $\mathrm{P}$, Yang J, Yu Q. MR imaging assessment of temporomandibular joint soft tissue injuries in dislocated and nondislocated mandibular condylar fractures. AJNR Am J Neuroradiol. 2009;30(1):59-63.

18. Sun W-H, Wang P-C. Temporomandibular joint capsule rupture presenting as expandable ear canal mass. J Formos Med Assoc. 2018;117(1):80-1.

19. Orhan K. Introduction to TMJ imaging. In: Rozylo-Kalinowska I, Orhan K. Imaging of the temporomandibular Suiza: Springer; 2018. p. 1-8.

20. Shi J, Xia J, Wei Y, et al. Three-dimensional virtual reality simulation of periarticular tumors using Dextroscope reconstruction and simulated surgery: A preliminary 10-case study. Med Sci Monit. 2014;20:1043-50.

21. Ahmad M, Hollender L, Anderson Q, et al. Research diagnostic criteria for temporomandibular disorders (RDC/TMD): Development of image analysis criteria and examiner reliability for image analysis. Oral Surg Oral Med Oral Pathol Oral Radiol Endod. 2009;107:844-60.

22. Yang Z, Wang M, Ma Y, et al. Magnetic resonance imaging (MRI) evaluation for anterior disc displacement of the temporomandibular joint. Med Sci Monit. 2017;23:712-8.

23. Agustsson $H$. Radiologic evaluation of the temporomandibular joint. En: McKinnins L. Fundamentals of Musculoskeletal Imaging. 4th edition. Pennsylvania, USA: F.A Davis Company; 2014. p. 212-67.

24. Pedullà E, Meli GA, Garufi A, et al. Morphometric evaluation of the temporomandibular joint and the masticatory spaces: the role of high-definition MRI. 
Minerva Stomatol. 2009;58(4):18.

25. Petscavage-Thomas JM, Walker EA. Unlocking the jaw: advanced imaging of the temporomandibular joint. AJR Am J Roentgenol. 2014;203(5):1047-58.

26. Tomas X, Pomes J, Berenguer J, et al. MR imaging of temporomandibular joint dysfunction: a pictorial review. RadioGraphics. 2006;26(3):765-81.

27. Roth C, Ward RJ, Tsai S, Zolotor W, Tello R. MR. MR imaging of the TMJ: a pictorial essay. Appl Radiol. 2005;34:9-16.

28. Orhan K, Aksoy S. Magnetic resonance imaging of TMJ. En: Rozylo-Kalinowska I, Orhan K. Imaging of the Temporomandibular. Suiza: Springer; 2018. p. 155-204.

29. Omami G. Magnetic resonance imaging of temporomandibular joint disc abnormalities: a pictorial essay. J Oral Maxillofac Radiol. 2013;1(3):99.

30. Aiken A, Bouloux G, Hudgins P. MR imaging of the temporomandibular joint. Magn Reson Imaging Clin N Am. 2012;20:397-412.

31. Alonso M, Gamba T, Pereira S, et al. Magnetic resonance imaging of the temporomandibular joint acquired using different parameters. J Morphol Sci. 2014;31(2):103-9.

32. Talmaceanu D, Lenghel LM, Hedesiu M, et al. Imaging modalities for temporomandibular joint disorders: an update. Clujul Med. 2018;91(3):280-7.

33. Al-Saleh MAQ, Punithakumar K, Lagravere M, Boulanger P, Jaremko JL, Major PW. Threedimensional assessment of temporomandibular joint using MRI-CBCT image registration. Cray JJ, editor. PLoS ONE. 2017;12(1):e0169555.

34. Kim BC, Lee YC, Cha HS, et al. Characteristics of temporomandibular joint structures after mandibular condyle fractures revealed by magnetic resonance imaging. Maxillofac Plast Reconstr Surg. 2016;38:24.

35. Sakhavalkar PU, Bhoosreddy AR, Kotwal HJ. Assessment and comparison of the capsular width of temporomandibular joint on ultrasonography and magnetic resonance imaging. J Indian Acad Oral Med Radiol. 2016;28:251-7.

36. Whaites E, Drage N. La imagen radiográfica. En: Whaites E, Drage N. Fundamentos de radiología dental. 5ta ed. Barcelona, España: Elsevier Mason; 2014. p. 1-12.

37. Westbrook C, Talbot J. Image weighting and contrast. En: Westbrook C. MRI in practice. 5ta ed. Oxford: Wiley Blackwell; 2019. p. 24-57.

38. Okeson PJ, Tamimi D. Introduction to MR imaging. In: Tamimi D, Hatcher D. Specialty Imaging Temporomandibular. Philadelphia: Elsevier; 2016. p. 346-53.

39. Cissel D. Quantitative MR of cartilage and implications for TMJ imaging. En: Tamimi D, Hatcher D. Specialty Imaging by. Philadelphia: Elsevier; 2016. p. 354-7.
40. Gatehouse PD, Bydder GM. Magnetic resonance imaging of short T2 components in tissue. Clin Radiol. 2003;58(1):1-19.

41. Cheng Q, Zhao F-C. Comparison of 1.5- and 3.0-T magnetic resonance imaging for evaluating lesions of the knee. Med Baltim. 2018;97(38):e12401.

42. Orhan K, Ucok O, Delilbasi C, Paksoy C, Karakurumer $\mathrm{K}$, Ozen T. Prevalence of temporomandibular joint sideways disc displacement in symptom-free volunteers and comparison of signal intensity ratios of masticator muscles on magnetic resonance images. OHDMBSC. 2005;4(4):14-8.

43. Hagenkord A, Basel CH. MRI imaging of the temporomandibular joint (TMJ) with regard to degeneration and disk displacement. Stockholm: European Society of Muscoloskeletal Radiology; 2015. DOI: 10.1594/ essr2015/P-0023

44. Shaefer J, Riley C, Caruso P, Keith D. Analysis of criteria for MRI diagnosis of TMJ disc displacement and arthralgia. Int J Dent. 2012;1-8.

45. Steenks MH, Bleys RL, Witkamp TD. Temporomandibular joint structures: a comparison between anatomic and magnetic resonance findings in a sagittal and an angulated plane. J Orofac Pain. 1994;8(2):120-35.

46. Chen YJ, Gallo LM, Meier D, Palla S. Individualized oblique-axial magnetic resonance imaging for improved visualization of mediolateral TMJ disc displacement. J Orofac Pain. 2000;14(2):128-39.

Recibido: 15-09-2019

Aceptado: 07-12-2019 